\title{
Natural Preservatives to Improve Food Quality and Safety
}

\author{
Moreno Bondi, ${ }^{1}$ Andrea Lauková, ${ }^{2}$ Simona de Niederhausern, ${ }^{1}$ \\ Patrizia Messi, ${ }^{1}$ and Chrissanthy Papadopoulou ${ }^{3}$ \\ ${ }^{1}$ Department of Life Sciences, University of Modena and Reggio Emilia, Via G. Campi 287, Modena, Italy \\ ${ }^{2}$ Institute of Animal Physiology, Slovak Academy of Sciences, Soltesovej 4-6, 04001 Kosice, Slovakia \\ ${ }^{3}$ Microbiology Department, Faculty of Medicine, School of Health Sciences, University of Ioannina, Ioannina, Greece
}

Correspondence should be addressed to Moreno Bondi; moreno.bondi@unimore.it

Received 12 October 2017; Accepted 12 October 2017; Published 12 December 2017

Copyright (C) 2017 Moreno Bondi et al. This is an open access article distributed under the Creative Commons Attribution License, which permits unrestricted use, distribution, and reproduction in any medium, provided the original work is properly cited.

Food products can be contaminated by a variety of pathogenic and spoilage microbiota, the former causing foodborne diseases and the latter causing significant economic losses for the food industry due to undesirable effects on the food properties. According to the Centers for Disease Control and Prevention (CDC), each year in the United States, 48 million people get sick, 128,000 are hospitalized, and 3,000 die because of foodborne infections. However, although foodborne outbreaks are well recorded, sporadic cases are not because not all patients visit the doctor or enter the hospital and the causative agent is not always identified. A considerable number of foodborne pathogens of great public health importance (e.g., VTEC E. coli serotypes, Campylobacter jejuni, Listeria monocytogenes, and Yersinia enterocolitica) have emerged during the recent two decades causing severe illness and foodborne outbreaks worldwide.

Microbial food spoilage is also an area of immense concern for the food industry. It is estimated that as much as $25 \%$ of all food produced is lost after harvest due to microbial activity. While the growth of spoilage microbiota in foods is not harmful for the human health, it has negative impact on the shelf-life, textural characteristics, and overall quality of the finished products, affects the consumer choices, and results in significant commercial losses. Thus, prevention or inhibition of microbial growth in foods is of outmost importance for the current globalized food production. Hence, there is still the need for new processing methods, to be used either alone or in combination with the already existing ones, able to reduce or eliminate foodborne pathogens and spoilage bacteria.

Chemical additives have been extensively used to prevent the survival and proliferation of microorganisms, but their safety and impact on human health are under discussion. Since the reduction or elimination of pathogens and spoilage microorganisms in food is the foremost priority, the current trends in food processing are focusing on the use of natural compounds, which are considered as safe alternatives and satisfy the consumer preferences for more "green foods." Hence the increased awareness on the safety of food additives and preservatives, and the consumer's trend to avoid foods containing chemicals, which, in the long-term, may have adverse impact on their health, have generated a significant number of studies and publications on the potential use of various natural substances, recognized as GRAS (Generally Recognized as Safe), to be used as food preservatives. Chemical compounds added to foods as antioxidant agents are also of concern, particularly when important protein sources (e.g., burgers, steaks) are involved in the daily diet of westernized consumer living in the developed countries [1,2]. Synthetic antioxidants such as BHT, TBHQ, and BHA have demonstrated various adverse effects on the human health including allergy, headache, asthma, and dermatitis. Recent studies on the utilization of natural antioxidants (e.g., herbal essential oils and extracts) indicate their capacity and safety $[3,4]$. Also, the antimicrobial properties of natural substances such as plant essential oils and extracts have been extensively studied with promising results [5]. The proper recycling and use of fruit-processing plants by-products like fruit seeds or skins discarded and piling up in huge amounts every year, are of great interest for the food industry, particularly their potential to be a useful source of oil and meal [6]. Additionally, the oil from plant seeds can be used by the food industry for manufacturing more "natural" or "green" foods and also can sufficiently extend the shelf-life of the 
food product [7]. The oils derived from fruit seeds oils may also have some more useful properties beyond being just "edible." The oil derived from the seed extract of two Mexican varieties of cactus pear (Opuntia albicarpa and Opuntia ficus indica) has been found to have sufficient antioxidant and antimicrobial properties [8]. Furthermore, some biological active compounds produced by microorganisms are widely used in the food industry as well. A good alternative to conventional chemically synthesized food preservatives is the use of natural antimicrobials such as bacteriocins produced by lactic acid bacteria (LAB), with Nisin being officially employed by the food industry and thus the most widely used. Bacteriocins are peptides, actually microbial toxins, produced from various microorganisms, and so far some of them (e.g., enterocins produced mostly by enterococci) have shown remarkable antimicrobial potential and their application as a natural barrier against pathogens and food spoilage has been proven to be very efficient, when used in the form of purified or semipurified extracts or as protective cultures $[9,10]$. Therefore, as the developments in food preservation are focusing on the implementation of natural antimicrobials and antioxidants this special issue explores the potential of alternatives to currently used preservatives through the publication of five high-quality articles, which aim to address recent advancements in the field of natural food preservatives and antioxidants. The prospect to replace synthetic preservatives with natural substances has been demonstrated by comparing the effects of Shirazi thyme, cinnamon, and rosemary extracts (denoted as natural antioxidants) with those of the synthetic antioxidant BHT on protein and lipid oxidations, physicochemical, microbial, and sensory characteristics of frozen beef burgers during storage. Specifically, the oxidative stability of the beef burgers containing Shirazi thyme, cinnamon, and rosemary extracts was well demonstrated, and the antibacterial activity was documented, as the total microbial counts of the tested burgers were well below the maximum allowed limit. Besides the evaluation of the antimicrobial and antioxidant properties of natural substances it is important to optimize their recovery. With regard to bacteriocins, to enhance Enterocin Y31 production and simplify the steps of separation and purification, a proper simplified and defined medium (SDM) has been developed for the Enterococcus faecium Y31 growth and enhancement of the Enterocin Y31 production. The bacterial growth did not result in Enterocin Y31 production in MRS medium and, therefore, both the growth rate and the Enterocin Y31 production were set as the goal for the investigation. Single omission experiments revealed that $5 \mathrm{~g} / \mathrm{L} \mathrm{NaCl}$, five vitamins, two nucleic acid bases, $\mathrm{MgSO} 4 \cdot 7 \mathrm{H} 2 \mathrm{O}, \mathrm{MnSO} 4 \cdot 4 \mathrm{H} 2 \mathrm{O}, \mathrm{KH} 2 \mathrm{PO} 4$, $\mathrm{K} 2 \mathrm{HPO} 4, \mathrm{CH} 3 \mathrm{COONa}$, fourteen amino acids, and glucose were essential for the adequate strain growth and Enterocin Y31 production. As a result, a novel simplified and defined medium (SDM) was formulated containing 30 ingredients, in which the Enterocin Y31 production yield was higher when compared to either MRS or CDM. The development of the SDM improved the Enterocin Y31 production and simplified the steps of purification (only two steps), which is very promising and increases its potential applications. Referring to seeds' oils, the optimization of the extraction conditions of cactus pear seed oil has been obtained using ultrasound in a closed system based on the antioxidant activity and using response surface methodology. The yield extraction and antioxidant and antimicrobial activity were compared with those obtained using both conventional and unconventional methods, such as Soxhlet and maceration, using heat, agitation or long extraction times, microwave, supercritical fluids and ultrasound-assisted extraction in an open and in a closed system. The results have shown that ultrasound exhibited lower oil yield and antioxidant activity but had the potential to achieve comparable results if multiple ultrasound extractions are performed in the time needed by conventional methods. Seed oils showed similar antimicrobial activity despite the extraction method and can be an alternative extraction method of seed oils from fruits such as cactus pear. Lastly, the replacement of chemical additives with natural compounds has been addressed for fish foods as well. Fish-paste products also known as fish cakes or surimi-based products are worldwide favorites. Surimi, a wet protein concentrate of fish muscle, is used as an intermediate raw material to produce surimi seafood. The flavor, texture, taste, shelf-life, and market value of surimi-based products depend on several factors, including the additives used to prepare the surimi. While preparing surimi with chemical additives, several problems have been observed, such as a lack of unique characteristics, inferior acceptability, and poor functionality. In this context, a systematic review of fishpaste products prepared using natural food additives (e.g., animal, seafood and plant source additives, herbs and oriental medicines, grains and roots, and functional food materials) has been performed, which summarizes the existing relevant knowledge in the production of new value-added foodstuffs of interest for the surimi industry.

In conclusion, this special issue offers to the readers the chance to be informed on the recent advancements related to the antimicrobial and antioxidant properties of natural substances that are of interest for the contemporary food industry. Their prospective use in the food production has the potential to lead towards the production of safer and healthier foods, not excluding their contribution to a more efficient preservation of the environment, when chemicals will be replaced with natural substances.

\section{Moreno Bondi \\ Andrea Lauková \\ Simona de Niederhausern \\ Patrizia Messi \\ Chrissanthy Papadopoulou}

\section{References}

[1] G. Comi, E. Tirloni, D. Andyanto, M. Manzano, and L. Iacumin, "Use of bio-protective cultures to improve the shelf-life and the sensorial characteristics of commercial hamburgers," LWT Food Science and Technology, vol. 62, no. 2, pp. 1198-1202, 2015.

[2] E. B. Özvural, Q. Huang, and M. L. Chikindas, "The comparison of quality and microbiological characteristic of hamburger patties enriched with green tea extract using three techniques: Direct addition, edible coating and encapsulation," LWT - Food Science and Technology, vol. 68, pp. 385-390, 2016. 
[3] S. Arabshahi-D, D. Vishalakshi Devi, and A. Urooj, "Evaluation of antioxidant activity of some plant extracts and their heat, $\mathrm{pH}$ and storage stability," Food Chemistry, vol. 100, no. 3, pp. 1100$1105,2007$.

[4] X. Zhang, L. Deyong, Q. Meng, C. He, and L. Ren, "Effect of mulberry leaf extracts on color, lipid oxidation, antioxidant enzyme activities and oxidative breakdown products of raw ground beef during refrigerated storage," Journal of Food Quality, 2016.

[5] H. Sakkas, P. Gousia, V. Economou, V. Sakkas, S. Petsios, and C. Papadopoulou, "In vitro antimicrobial activity of five essential oils on multidrug resistant Gram-negative clinical isolates," Journal of Intercultural Ethnopharmacology, vol. 5, no. 3, pp. 212-218, 2016.

[6] B. Kamel and Y. Kakuda, "Fatty acids in fruits and fruit products," in Fatty Acids in Foods and their Health Implications, Third Edition, vol. 20073230 of Food Science and Technology, pp. 263301, CRC Press, 3rd Edition edition, 2007.

[7] F. Solórzano-Santos and M. G. Miranda-Novales, "Essential oils from aromatic herbs as antimicrobial agents," Current Opinion in Biotechnology, vol. 23, no. 2, pp. 136-141, 2012.

[8] P. Zito, M. Sajeva, M. Bruno, S. Rosselli, A. Maggio, and F. Senatore, "Essential oils composition of two Sicilian cultivars of Opuntia ficus-indica (L.) Mill. (Cactaceae) fruits (prickly pear)," Natural Product Research (Formerly Natural Product Letters), vol. 27, no. 14, pp. 1305-1314, 2013.

[9] R. Iseppi, F. Pilati, M. Marini et al., "Anti-listerial activity of a polymeric film coated with hybrid coatings doped with Enterocin $416 \mathrm{~K} 1$ for use as bioactive food packaging," International Journal of Food Microbiology, vol. 123, no. 3, pp. 281-287, 2008.

[10] A. Lauková and P. Turek, "Effect of enterocin 4231 in Slovak fermented salami púchov after its experimental inoculation with Listeria Innocua Li," ACTA Scientiarum Polonorum Technologia Alimentaria, vol. 10, no. 4, pp. 423-431, 2011. 

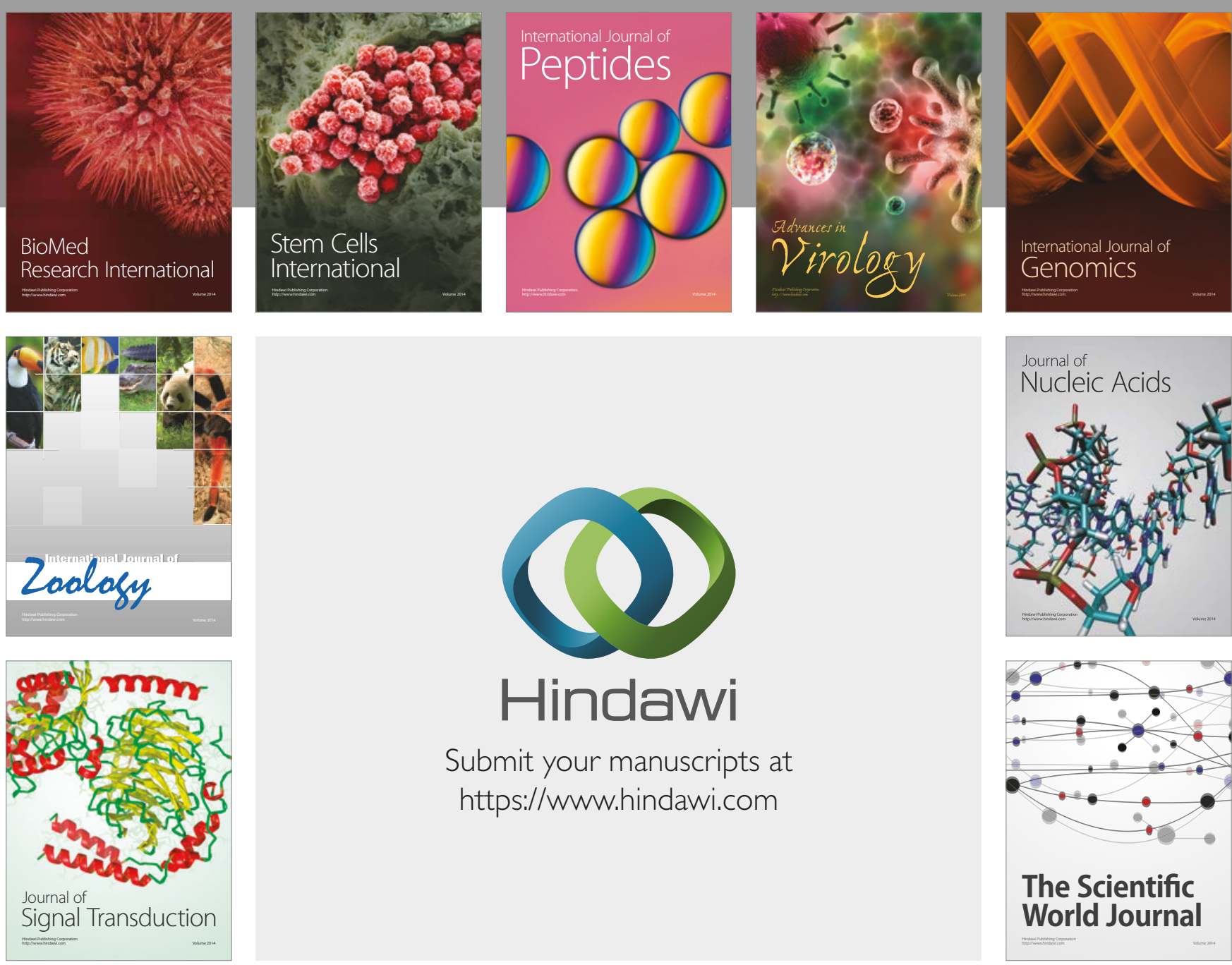

Submit your manuscripts at

https://www.hindawi.com
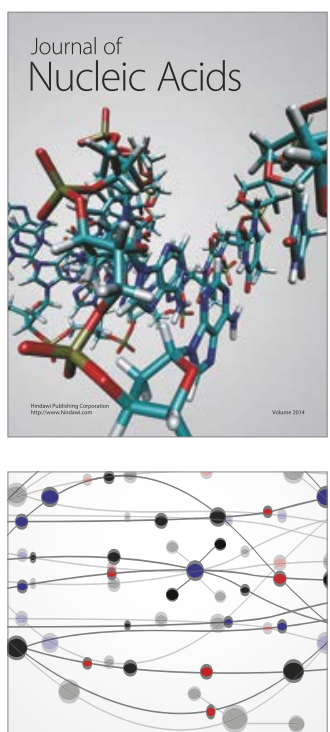

The Scientific World Journal

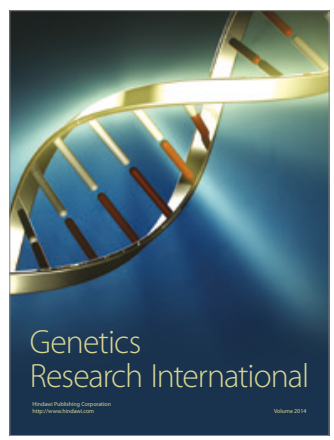

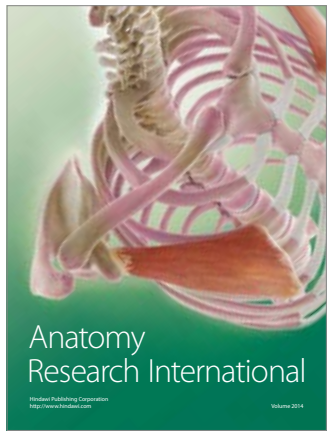

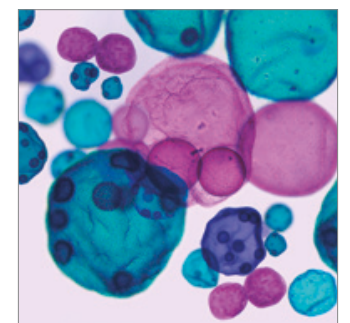

International Journal of Microbiology
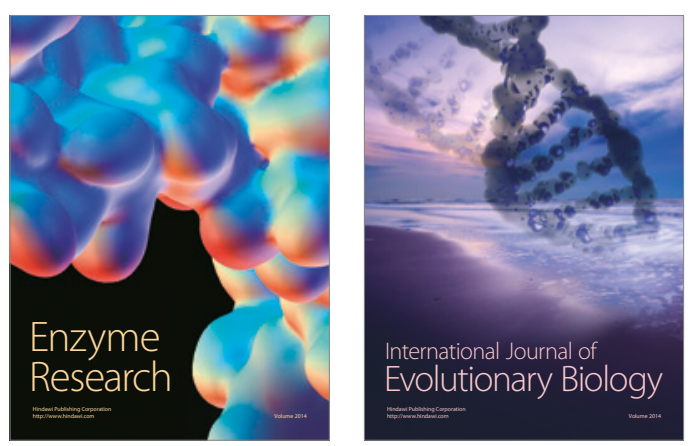
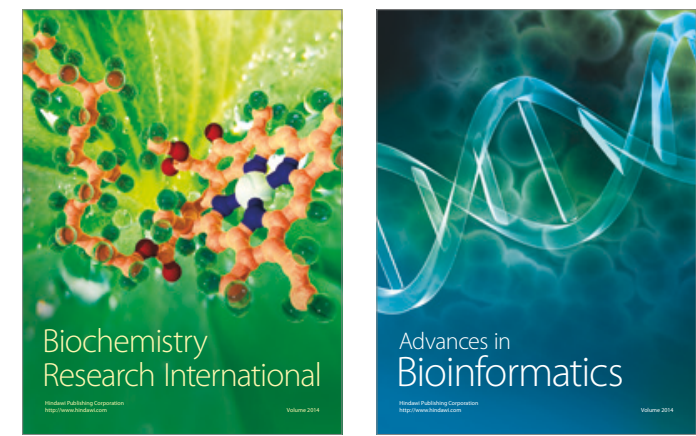

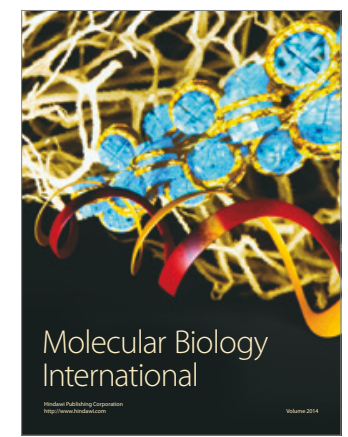

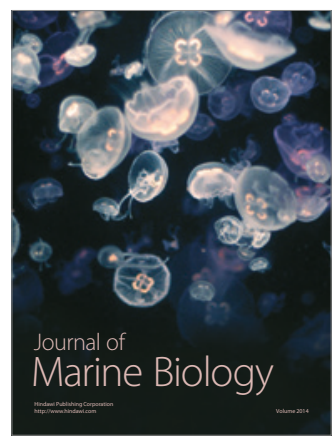

